\title{
Extremal Properties of the Zagreb Eccentricity Indices
}

\author{
Zhibin Du, ${ }^{a}$ Bo Zhou, ${ }^{\text {b,* }}$ and Nenad Trinajsticic \\ ${ }^{a}$ Department of Mathematics, Tongji University, Shanghai 200092, China \\ ${ }^{\mathrm{b}}$ Department of Mathematics, South China Normal University, Guangzhou 510631, China \\ ${ }^{\mathrm{c}}$ The Rugjer Bošković Institute, P. O. Box 180, HR-10002 Zagreb, Croatia
}

RECEIVED NOVEMBER 17, 2011; ACCEPTED SEPTEMBER 28, 2012

\begin{abstract}
We give sharp lower bounds for the Zagreb eccentricity indices of connected graphs with fixed numbers of vertices and edges, sharp lower and upper bounds for the Zagreb eccentricity indices of trees with fixed number of pendant vertices, sharp upper bounds for the Zagreb eccentricity indices of trees with fixed matching number (fixed maximum degree, respectively), and characterize the extremal graphs. (doi: 10.5562/cca2020)
\end{abstract}

Keywords: Zagreb eccentricity indices, trees, pendant vertices, matching number, maximum degree

\section{INTRODUCTION}

Let $G$ be a connected graph with vertex set $V(G)$ and edge set $E(G)$. For a vertex $u \in V(G), d_{G}(u)$ or $d_{u}$ denotes the degree of $u$ in $G .^{1,2}$ The first Zagreb index of $G$ is defined as: ${ }^{3,4}$

$$
M_{1}(G)=\sum_{u \in V(G)} d_{u}^{2},
$$

while the second Zagreb index of $G$ is defined as: ${ }^{3,4}$

$$
M_{2}(G)=\sum_{u v \in E(G)} d_{u} d_{v}
$$

The properties of these molecular indices and their derivatives are continuously studied, e.g., in Reference 5. Let us also point to the timely paper that just appeared in this journal by Stevanović ${ }^{6}$ reporting on the relationship between these two descriptors, a topic that is also in recent years considerably studied, e.g., in References 7 and 8. It should be also pointed out that the Zagreb indices and their variants are useful molecular descriptors which found considerable use in QSPR and QSAR studies as summarized by Todeschini and Consonni. ${ }^{9,10}$

For a vertex $u \in V(G), e_{G}(u)$ or $e_{u}$ denotes the eccentricity of $u$ in $G$, which is equal to the largest distance from $u$ to other vertices. ${ }^{1,2}$ The Zagreb eccentricity indices were introduced in an analogous way as the Zagreb indices by Vukičević and Graovac. ${ }^{11}$ The first Zagreb eccentricity index of $G$ is defined as:

$$
\xi_{1}(G)=\sum_{u \in V(G)} e_{u}^{2}
$$

while the second Zagreb eccentricity index of $G$ is defined as:

$$
\xi_{2}(G)=\sum_{u v \in E(G)} e_{u} e_{v}
$$

Vukičević and Graovac ${ }^{11}$ studied the comparison of $\frac{\xi_{1}(G)}{n}$ and $\frac{\xi_{2}(G)}{m}$ for a graph $G$ with $n$ vertices and $m$ edges. Some mathematical and computational properties of the Zagreb eccentricity indices have recently been established, ${ }^{12}$ where the authors gave lower and upper bounds for the Zagreb eccentricity indices of connected graphs in terms of graph invariants such as the number of vertices, the number of edges, the radius, and the diameter, determined the $n$-vertex trees with the first a few smallest and largest Zagreb eccentricity indices for $n \geq 6$, and found lower and upper bounds for the Zagreb eccentricity indices of trees with fixed diameter, lower bounds for the Zagreb eccentricity indices of trees with fixed matching number.

In continuation to our study reported in Reference 12 , in this paper, we give sharp lower bounds for the Zagreb eccentricity indices of connected graphs with fixed numbers of vertices and edges, sharp lower and upper bounds for the Zagreb eccentricity indices of trees with fixed number of pendant vertices, sharp upper bounds for the Zagreb eccentricity indices of trees with

\footnotetext{
* Author to whom correspondence should be addressed. (E-mail: zhoubo@scnu.edu.cn)
} 
fixed matching number (fixed maximum degree, respectively), and characterize the extremal graphs.

\section{PRELIMINARIES}

Let $K_{n}$ and $P_{n}$ be, respectively, the complete graph and the path with $n$ vertices. ${ }^{1,2}$ By direct calculation, if $n \geq 2$ is even, then: ${ }^{12}$

$$
\begin{aligned}
& \xi_{1}\left(P_{n}\right)=\frac{7 n^{3}-9 n^{2}+2 n}{12}, \\
& \xi_{2}\left(P_{n}\right)=\frac{7 n^{3}-21 n^{2}+20 n}{12},
\end{aligned}
$$

and if $n \geq 3$ is odd, then: ${ }^{12}$

$$
\begin{aligned}
& \xi_{1}\left(P_{n}\right)=\frac{7 n^{3}-9 n^{2}-n+3}{12}, \\
& \xi_{2}\left(P_{n}\right)=\frac{7 n^{3}-21 n^{2}+17 n-3}{12} .
\end{aligned}
$$

Let $G_{1}$ and $G_{2}$ be the trees shown in Figure 1, where vertices $u$ and $v$ are adjacent, $u$ has a unique neighbor in $N$. In $G_{1}, u$ has at least one neighbor in $M$, and all of such neighbors are switched to be neighbors of $v$ in $G_{2}$.

Lemma 1. Let $G_{1}$ and $G_{2}$ be the trees shown in Figure 1 .

(i) If there is a diametrical path of $G_{1}$ with all edges in $E(Q)$, then $\xi_{1}\left(G_{1}\right)>\xi_{1}\left(G_{2}\right)$ and $\xi_{2}\left(G_{1}\right)>$ $\xi_{2}\left(G_{2}\right)$.

(ii) If there is a diametrical path of $G_{1}$ containing vertex $u$ and some vertices in $N$ and $Q$, and $e_{G_{1}}(u) \geq e_{G_{1}}(v)$, then $\xi_{1}\left(G_{1}\right) \geq \xi_{1}\left(G_{2}\right)$ and $\xi_{2}\left(G_{1}\right) \geq$ $\xi_{2}\left(G_{2}\right)$ with either equality if and only if $e_{G_{1}}(u)=e_{G_{1}}(v)$.

Remark 1. In Lemma 1 (i) $u$ is a vertex with degree at least three outside a diametrical path of $G_{1}$, while in Lemma 1 (ii) $u$ is a vertex with degree at least three in a diametrical path of $G_{1}$.

Let $\widetilde{\sigma}(n, p)$ be the set of trees with $n$ vertices and $p$ pendant vertices, where $2 \leq p \leq n-1$. The cases $p=2, n-1$ are trivial.
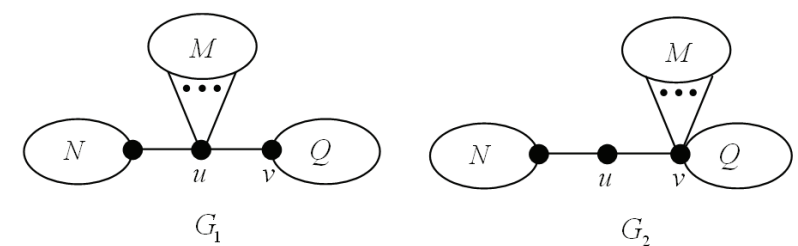

Figure 1. Trees $G_{1}$ and $G_{2}$ in Lemma 1.
Lemma 2. Let $G$ be a tree in $\mathscr{T}(n, p)$ with minimum Zagreb eccentricity indices, where $3 \leq p \leq n-1$. Let $P=v_{0} v_{1} \ldots v_{d}$ be a diametrical path of $G$. Then the vertices with degree at least three in $G$ can only be the central vertices of $P$.

For integers $n$ and $p$ with $3 \leq p \leq n-2$, let $k=\left\lfloor\frac{n-1}{p}\right\rfloor$ and $r=n-1-k p$, let $T_{1}^{(n, p)}$ be the tree obtained by attaching $p-r$ paths on $k$ vertices and $r$ paths on $k+1$ vertices to a common vertex, and if $n-2 \equiv 0(\bmod p)$, then let $T_{2}^{(n, p)}(s)$ be the tree obtained by attaching $s$ paths and $p-s$ paths on $\frac{n-2}{p}$ vertices, respectively, to the two end vertices of an edge, where $1 \leq s \leq\lfloor p / 2\rfloor$. Obviously, $T_{2}^{(n, p)}(1) \cong T_{1}^{(n, p)}$ if $n-2 \equiv 0(\bmod p)$.

Lemma 3. Let $G \in \mathscr{T}(n, p)$, where $3 \leq p \leq n-2$. If $G$ has exactly one vertex with degree at least three, then $\xi_{1}\left(G_{1}\right) \geq \xi_{1}\left(T_{1}^{(n, p)}\right)$ and $\xi_{2}\left(G_{1}\right) \geq \xi_{2}\left(T_{1}^{(n, p)}\right)$ with either equality if and only if $G \cong T_{1}^{(n, p)}$.

Let $T_{a}^{n, s}$ be the ( $n$-vertex) tree obtained by attaching $a$ and $s-a$ pendant vertices, respectively, to the two end vertices of the path $P_{n-s}$, where $1 \leq a \leq\lfloor s / 2\rfloor$. Let $\mathbf{T}^{n, s}=\left\{T_{a}^{n, s}: 1 \leq a \leq\lfloor s / 2]\right\}$.

For integers $d$ and $n$ with $2 \leq d \leq n-1$, let

$$
\begin{aligned}
& f_{1}(n, d)= \begin{cases}d^{2} n-\frac{5 d^{3}-2 d}{12} & \text { if } d \text { is even } \\
d^{2} n-\frac{5 d^{3}-5 d}{12} & \text { if } d \text { is odd }\end{cases} \\
& f_{2}(n, d)= \begin{cases}d(d-1) n-\frac{5 d^{3}-8 d}{12} & \text { if } d \text { is even } \\
d(d-1) n-\frac{5 d^{3}-11 d-6}{12} & \text { if } d \text { is odd. }\end{cases}
\end{aligned}
$$

Lemma $4 .{ }^{12}$ Let $G$ be a tree with $n$ vertices and diameter $d$. Then:

$$
\begin{aligned}
& \xi_{1}(G) \leq f_{1}(n, d) \\
& \xi_{2}(G) \leq f_{2}(n, d)
\end{aligned}
$$

with either equality if and only if $G \in \mathbf{T}^{n, n-d+1}$. For $2 \leq d \leq n-2$, we have:

$$
\begin{aligned}
& f_{1}(n, d)<f_{1}(n, d+1) \\
& f_{2}(n, d)<f_{2}(n, d+1) .
\end{aligned}
$$


Recall that a pendant path at a vetex $v$ of a tree $T$ is a path in which no vetex other than $v$ lies in any edges of $T$ off the path, where the degree of $v$ is at least three.

\section{BOUNDS AND EXTREMAL GRAPHS}

Denote by $G \vee H$ the graph obtained from vertexdisjoint graphs $G$ and $H$ by adding edges between each vertex in $G$ and each vertex in $H$. For positive integers $n$ and $m$ with $n-1 \leq m<\left(\begin{array}{l}n \\ 2\end{array}\right)$, let $a=a_{n, m}=$ $\left\lfloor\frac{2 n-1-\sqrt{(2 n-1)^{2}-8 m}}{2}\right\rfloor$, and $\mathbf{G}_{(n, m)}$ be the set of graphs $K_{a} \vee H$, where $H$ is a graph with $n-a$ vertices and $m-\left(\begin{array}{l}a \\ 2\end{array}\right)-a(n-a)$ edges. Note that $a$ with $1 \leq$ $a<n$ is the largest integer satisfying $2 m \geq a(n-1)+$ $a(n-a)$, i.e., $h(a) \geq 0$ with $h(a)=a^{2}-2 n a+a+2 m$, we have $\left[m-\left(\begin{array}{l}a \\ 2\end{array}\right)-a(n-a)\right]-(n-a-1)=\frac{1}{2} h(a+1)<$ 0 . This implies that each vertex of $H$ has eccentricity two in $K_{a} \vee H$.

Proposition 1. Let $G$ be an n-vertex connected graph with $m$ edges, where $n-1 \leq m<\left(\begin{array}{l}n \\ 2\end{array}\right)$. Let $a=a_{n, m}=$

$$
\begin{aligned}
& \left\lfloor\frac{2 n-1-\sqrt{(2 n-1)^{2}-8 m}}{2}\right\rfloor . \text { Then: } \\
& \xi_{1}(G) \geq 4 n-3 a \\
& \xi_{2}(G) \geq 4 m-3\left(\begin{array}{l}
a \\
2
\end{array}\right)-2 a(n-a)
\end{aligned}
$$

with either equality if and only if $G \in \mathbf{G}_{(n, m)}$.

Clearly, $a_{n, n}=1$ for $n \geq 4$, and $a_{n, n+1}=1$ for $n \geq 5$.

Corollary 1. Let $G$ be a unicyclic graph with $n \geq 4$ vertices. Then:

$$
\begin{aligned}
& \xi_{1}(G) \geq 4 n-3 \\
& \xi_{2}(G) \geq 2 n+2
\end{aligned}
$$

with either equality if and only if $G$ is the graph obtained by adding an edge to the star $S_{n}$.

Corollary 2. Let $G$ be a bicyclic graph with $n \geq 5$ vertices. Then:

$$
\begin{aligned}
& \xi_{1}(G) \geq 4 n-3 \\
& \xi_{2}(G) \geq 2 n+6
\end{aligned}
$$

with either equality if and only if $G$ is a graph obtained by adding two edges to the star $S_{n}$.

Proposition 2. Let $G \in \mathscr{T}(n, p)$, where $3 \leq p \leq n-2$. Let $k=\left\lfloor\frac{n-1}{p}\right\rfloor$ and $r=n-1-k p$. Then:

$\xi_{1}(G) \geq \begin{cases}\frac{p k}{6}(7 k+1)(2 k+1)+k^{2} & \text { if } r=0 \\ \frac{p k}{6}(14 k+13)(k+1)+2(k+1)^{2} & \text { if } r=1 \\ \frac{p k}{6}(14 k+13)(k+1)+(4 r+1)(k+1)^{2} & \text { if } r \geq 2\end{cases}$

$\xi_{2}(G) \geq \begin{cases}\frac{p k}{3}\left(7 k^{2}-1\right) & \text { if } r=0 \\ \frac{p k}{3}(7 k+2)(k+1)+(k+1)^{2} & \text { if } r=1 \\ \frac{p k}{3}(7 k+2)(k+1)+2 r(k+1)(2 k+1) & \text { if } r \geq 2\end{cases}$

with either equality if and only if $G \cong T_{1}^{(n, p)}$ or $G \cong T_{2}^{(n, p)}(s)$ with $2 \leq s \leq\lfloor p / 2\rfloor$ if $n-2 \equiv 0(\bmod p)$, and $G \cong T_{1}^{(n, p)}$ otherwise.

Proposition 3. Let $G \in \mathscr{T}(n, p)$, where $2 \leq p \leq n-1$. Then:

$\xi_{1}(G) \leq \begin{cases}(n-p+1)^{2} n-\frac{5(n-p+1)^{3}-2(n-p+1)}{12} & \text { if } n-p \text { is odd } \\ (n-p+1)^{2} n-\frac{5(n-p+1)^{3}-5(n-p+1)}{12} & \text { if } n-p \text { is even }\end{cases}$

$\xi_{2}(G) \leq \begin{cases}(n-p+1)(n-p) n-\frac{5(n-p+1)^{3}-8(n-p+1)}{12} & \text { if } n-p \text { is odd } \\ (n-p+1)(n-p) n-\frac{5(n-p+1)^{3}-11(n-p+1)-6}{12} & \text { if } n-p \text { is even }\end{cases}$

with either equality if and only if $G \in \mathbf{T}^{n, p}$.

Recall that the matching number of a graph $G$ is the maximum number of edges of matchings in $G .^{2}$ For $2 \leq \beta \leq\lfloor n / 2\rfloor$, the tree with minimum Zagreb eccentricity indices among trees with $n$ vertices and matching number $\beta$ has been determined. ${ }^{12}$

Proposition 4. Let $G$ be an $n$-vertex tree with matching number $\beta$, where $2 \leq \beta \leq\lfloor n / 2\rfloor$. Then: 


$$
\begin{aligned}
& \xi_{1}(G) \leq 4 \beta^{2} n-\frac{10 \beta^{3}-\beta}{3} \\
& \xi_{2}(G) \leq(4 \beta-2) \beta n-\frac{10 \beta^{3}-4 \beta}{3}
\end{aligned}
$$

with either equality if and only if $G \in \mathbf{T}^{n, n-2 \beta+1}$.

Proposition 5. Let $G$ be an n-vertex tree with maximum degree $\Delta$, where $2 \leq \Delta \leq n-1$. Then:

$\xi_{1}(G) \leq \begin{cases}(n-\Delta+1)^{2} n-\frac{5(n-\Delta+1)^{3}-2(n-\Delta+1)}{12} & \text { if } n-\Delta \text { is odd } \\ (n-\Delta+1)^{2} n-\frac{5(n-\Delta+1)^{3}-5(n-\Delta+1)}{12} & \text { if } n-\Delta \text { is even }\end{cases}$

$\xi_{2}(G) \leq \begin{cases}(n-\Delta+1)(n-\Delta) n-\frac{5(n-\Delta+1)^{3}-8(n-\Delta+1)}{12} & \text { if } n-\Delta \text { is odd } \\ (n-\Delta+1)(n-\Delta) n-\frac{5(n-\Delta+1)^{3}-11(n-\Delta+1)-6}{12} & \text { if } n-\Delta \text { is even }\end{cases}$

with either equality if and only if $G \cong T_{1}^{n, \Delta}$.

Supplementary Materials. - Supporting informations to the paper are enclosed to the electronic version of the article. These data can be found on the website of Croatica Chemica Acta (http://hrcak.srce.hr/cca)
Acknowledgements. This work was supported by the Guangdong Provincial Natural Science Foundation of China (Grant No. S2011010005539) and the Ministry of Science, Education and Sports of Croatia (Grant No. 098-1770945-2919).

\section{REFERENCES}

1. F. Harary, Graph Theory, 2nd printing, Addison-Wesley, Reading, MA, 1971, p. 35.

2. N. Trinajstić, Chemical Graph Theory, $2^{\text {nd }}$ revised edition, CRC press, Boca Raton, 1992.

3. I. Gutman and N. Trinajstić, Chem. Phys. Lett. 17 (1972) 535538.

4. I. Gutman, B. Ruščić, N. Trinajstić, and C. F. Wilcox, Jr., J. Chem. Phys. 62 (1975) 3399-3405.

5. S. Nikolić, G. Kovačević, A. Miličević, and N. Trinajstić, Croat. Chem. Acta 76 (2003) 113-124.

6. S. Stevanović, Croat. Chem. Acta 84 (2011) 17-19.

7. P. Hansen and D. Vukičević, Croat. Chem. Acta 80 (2007) 165-168.

8. A. Ilić and D. Stevanović, MATCH Commun. Math. Comput. Chem. 62 (2009) 681-687.

9. R. Todeschini and V. Consonni, Handbook of Molecular Descriptors, Wiley-VCH, Weinheim, 2000, p. 124.

10. R. Todeschini and V. Consonni, Molecular Descriptors for Chemoinformatics, Wiley-VCH, Weinheim, Vol. I, 2009, pp. 237-241.

11. D. Vukičević and A. Graovac, Acta Chim. Slov. 57 (2010) 524-538.

12. R. Xing, B. Zhou, and N. Trinajstić, Croat. Chem. Acta $\mathbf{8 4}$ (2011) 493-497. 


\section{SUPPLEMENTARY MATERIALS}

\section{Proof of Lemma 1}

Note that for a diametrical path $P$ of $G_{1}$ and $x \in V\left(G_{1}\right)$, there is a pendant vertex, say $y$, in $P$ such that the distance from $x$ to $y$ in $G_{1}$ is equal to $e_{G_{1}}(x)$. Note that $e_{G_{1}}(x)=e_{G_{2}}(x)$ for $x \in V\left(G_{1}\right) \backslash V(M)$ and $e_{G_{1}}(x)-e_{G_{2}}(x)=e_{G_{1}}(u)-e_{G_{2}}(v)=e_{G_{1}}(u)-e_{G_{1}}(v)$ for $x \in V(M)$ in (i) and (ii), while $e_{G_{1}}(u)>e_{G_{1}}(v)$ in (i) and $e_{G_{1}}(u) \geq e_{G_{1}}(v)$ in (ii). Then the result follows easily.

\section{Proof of Lemma 2}

The cases $d=2,3$ are obvious. Suppose that $d \geq 4$. By Lemma 1 (i) and Remark 1, there is no vertex in $G$ outside $P$ with degree at least three. Now we show that the vertices with degree at least three on $P$ can only be the central vertices of $P$.

Let $v_{i}$ with $1 \leq i \leq d-1$ be a vertex on $P$ with degree at least three. If $e_{G}\left(v_{i}\right)>e_{G}\left(v_{i+1}\right)\left(e_{G}\left(v_{i}\right)>e_{G}\left(v_{i-1}\right)\right.$, respectively), then making use of Lemma 1 (ii) to $G_{1}=G$ by setting $u=v_{i}$ and $v=v_{i+1}\left(v=v_{i-1}\right.$, respectively), we may get another tree in $\mathscr{T}(n, p)$ with smaller Zagreb eccentricity indices, a contradiction. Thus $e_{G}\left(v_{i}\right) \leq e_{G}\left(v_{i+1}\right)$ and $e_{G}\left(v_{i}\right) \leq e_{G}\left(v_{i-1}\right)$. Obviously, $e_{G}\left(v_{i}\right)=d-i$ or $i$. In the former case, $i \leq d-i, d-i \leq i+1$ since $e_{G}\left(v_{i}\right) \leq e_{G}\left(v_{i+1}\right)$, and thus $2 i=d-1$ or $d$. In the latter case, $i \geq d-i, i \leq d-i+1$ since $e_{G}\left(v_{i}\right) \leq e_{G}\left(v_{i-1}\right)$, and thus $2 i=d$ or $d+1$. Thus $2 i=d-1, d$ or $d+1$, i.e., $v_{i}$ is a central vertex of $P$.

\section{Proof of Lemma 3}

Let $G$ be a tree with minimum Zagreb eccentricity indices satisfying the given condition. Then there are $p$ pendant paths at a common vertex, say $v$. Among these pendant paths, let $Q_{1}$ be a path with maximum length $a, Q_{2}$ a path with maximum length $b$ except the path $Q_{1}$, and $Q_{3}$ a path with minimum length $c$, where $a \geq b \geq c$. Let $v_{1}$ be the neighbor of $v$ in $Q_{1}$. If $a>b+1$, then $e_{G}\left(v_{1}\right)<e_{G}(v)$, and thus making use of Lemma 1 (ii) to $G_{1}=G$ by setting $u=v$ and $v=v_{1}$, we may get another tree (also satisfying the given condition) with smaller Zagreb eccentricity indices, a contradiction. Thus $a=b$ or $b+1$. Suppose that $a \geq c+2$. Let $v_{2}$ ( $v_{3}$, respectively) be the pendant vertex in $Q_{1}\left(Q_{3}\right.$, respectively), and $v_{4}$ the neighbor of $v_{2}$. Let $G_{1}$ be the tree obtained from $G$ by deleting the edge $v_{2} v_{4}$ and adding the edge $v_{2} v_{3}$. Obviously, $G_{1} \in \mathscr{T}(n, p)$, and $G_{1}$ has exactly one vertex with degree at least three. Then $e_{G_{1}}(x) \leq e_{G}(x)$ for $x \in V(G), e_{G_{1}}\left(v_{2}\right)=b+c+1<a+b=e_{G}\left(v_{2}\right)$, and $e_{G_{1}}\left(v_{3}\right)=b+c<a+b-1=e_{G}\left(v_{4}\right)$. Thus $\xi_{1}(G)>\xi_{1}\left(G_{1}\right)$ and $\xi_{2}(G)>\xi_{2}\left(G_{1}\right)$, a contradiction. It follows that $a=c$ or $c+1$, i.e., $G \cong T_{1}^{(n, p)}$.

\section{Proof of Proposition 1}

Note that $1 \leq a<n$. Let $k$ be the number of vertices with degree $n-1$ in $G$, where $0 \leq k \leq n-2$. If $k=0$, then $\xi_{1}(G) \geq 4 n>4 n-3 a$ and $\xi_{2}(G) \geq 4 m>4 m-3\left(\begin{array}{l}a \\ 2\end{array}\right)-2 a(n-a)$. Suppose that $k \geq 1$. Then all the $n-k$ vertices of degree less than $n-1$ have eccentricity two, and thus:

$$
\begin{aligned}
& \xi_{1}(G)=1^{2} \cdot k+2^{2} \cdot(n-k)=4 n-3 k, \\
& \xi_{2}(G)=1 \cdot 1 \cdot\left(\begin{array}{l}
k \\
2
\end{array}\right)+1 \cdot 2 \cdot k(n-k)+2 \cdot 2 \cdot\left(m-\left(\begin{array}{l}
k \\
2
\end{array}\right)-k(n-k)\right)=4 m-3\left(\begin{array}{l}
k \\
2
\end{array}\right)-2 k(n-k) .
\end{aligned}
$$

Note that $2 m \geq k(n-1)+k(n-k)$ and recall that $a$ is the largest integer satisfying $2 m \geq a(n-1)+a(n-a)$, implying that $k \leq a$. Note that both $4 n-3 k$ and $4 m-3\left(\begin{array}{l}k \\ 2\end{array}\right)-2 k(n-k)$ are decreasing on $k$, and thus $\xi_{1}(G) \geq 4 n-3 a$ and $\xi_{2}(G) \geq 4 m-3\left(\begin{array}{l}a \\ 2\end{array}\right)-2 a(n-a)$ with either equality if and only if $G$ has exactly $a$ vertices of degree $n-1$ and all other vertices have eccentricity two, i.e., $G \in \mathbf{G}_{(n, m)}$.

\section{Proof of Proposition 2}

Let $G$ be a tree in $\mathscr{T}(n, p)$ with minimum Zagreb eccentricity indices. Let $V_{1}(G)$ be the set of vertices in $G$ with degree at least three. By Lemma $2,\left|V_{1}(G)\right|=1$ or 2 . If $\left|V_{1}(G)\right|=1$, then by Lemma 3, we have $G \cong T_{1}^{(n, p)}$. Suppose 
that $\left|V_{1}(G)\right|=2$. Let $V_{1}(G)=\{u, v\}$, and $P$ be a diametrical path of $G$. By Lemma $2, u, v$ are adjacent in $P$, and $e_{G}(u)=e_{G}(v)$. Among the pendant paths at $u$, let $k_{1}$ and $k_{2}$ be, respectively, the maximum and minimum length of them. Suppose that $k_{1}>k_{2}$. Obviously, the pendant path at $u$ with all vertices on $P$ is of length $k_{1}$. Let $G_{1}$ be the tree obtained from $G$ by switching all neighbors of $u$ in $G$ outside $P$ to be neighbors of $v$ in $G_{1}$. By Lemma 1 (ii), we have $\xi_{1}(G)=\xi_{1}\left(G_{1}\right)$ and $\xi_{2}(G)=\xi_{2}\left(G_{1}\right)$. Note that $\left|V_{1}\left(G_{1}\right)\right|=1$, and there are two pendant paths with lengths $k_{1}+1$ and $k_{2}$ at $v$ in $G_{1}$. Since $k_{1}+1-k_{2}>1$, by Lemma $3, \quad \xi_{1}(G)=\xi_{1}\left(G_{1}\right)>\xi_{1}\left(T_{1}^{(n, p)}\right)$ and $\xi_{2}(G)=\xi_{2}\left(G_{1}\right)>$ $\xi_{2}\left(T_{1}^{(n, p)}\right)$, a contradiction. Thus, all pendant paths at $u$ in $G$ have the same length. Similarly, all pendant paths at $v$ in $G$ have the same length. It follows that $G \cong T_{2}^{(n, p)}(s)$ with $2 \leq s \leq\lfloor p / 2\rfloor$.

\section{Proof of Proposition 3}

Let $d$ be the diameter of $G$. Note that there are $d-1$ non-pendant vertices on a diametrical path of $G$, and thus $p \leq n-(d-1)$, i.e., $d \leq n-p+1$. By Lemma $4, \quad \xi_{1}(G) \leq f_{1}(n, d) \leq f_{1}(n, n-p+1) \quad$ and $\quad \xi_{2}(G) \leq f_{2}(n, d) \leq$ $f_{2}(n, n-p+1)$. Obviously, $\xi_{1}(G)=f_{1}(n, n-p+1)$ and $\xi_{2}(G)=f_{2}(n, n-p+1)$ if and only if $G \in \mathbf{T}^{n, p}$.

\section{Proof of Proposition 4}

Let $d$ be the diameter of $G$. If $d \geq 2 \beta+1$, then we may construct a matching with $\beta+1$ edges by choosing pairwise disjoint edges on a diametrial path of $G$, a contradiction. Thus $d \leq 2 \beta$. By Lemma $4, \xi_{1}(G) \leq f_{1}(n, d) \leq$ $f_{1}(n, 2 \beta)$ and $\xi_{2}(G) \leq f_{2}(n, d) \leq f_{2}(n, 2 \beta)$. Obviously, $\xi_{1}(G)=f_{1}(n, 2 \beta)$ and $\xi_{2}(G)=f_{2}(n, 2 \beta)$ if and only if $G \in \mathbf{T}^{n, n-2 \beta+1}$.

\section{Proof of Proposition 5}

Let $d$ be the diameter of $G$, and $p$ be the number of pendant vertices of $G$. Recall that $d \leq n-p+1$ and note that $\Delta \leq p, \quad$ and thus $d \leq n-\Delta+1$. By Lemma $4, \quad \xi_{1}(G) \leq f_{1}(n, d) \leq f_{1}(n, n-\Delta+1) \quad$ and $\quad \xi_{2}(G) \leq f_{2}(n, d) \leq$ $f_{2}(n, n-\Delta+1)$. Obviously, $\xi_{1}(G)=f_{1}(n, n-\Delta+1)$ and $\xi_{2}(G)=f_{2}(n, n-\Delta+1)$ if and only if $G \cong T_{1}^{n, \Delta}$. 